\title{
A Narrow Bicontinuous Microemulsion Domain in Mixed Polymeric Silicone Systems
}

\author{
Suraj Chandra Sharma ${ }^{1}$, Koji Tsuchiya ${ }^{1}$, Kenichi Sakai ${ }^{1}$, Hideki Sakai ${ }^{1,2}$, \\ Masahiko Abe A $^{1,2}$ and Reiji Miyahara ${ }^{3 *}$ \\ ${ }^{1}$ Department of Pure and Applied Chemistry, Faculty of Science and Technology, Tokyo University of Science (2641 Yamazaki, Noda, Chiba \\ 278-8510, JAPAN) \\ ${ }^{2}$ Institute of Colloid and Interface Science, Tokyo University of Science (1-3 Kagurazaka, Shinjuku, Tokyo 162-8601, JAPAN) \\ ${ }^{3}$ Shiseido Research Center (2-2-1 Hayabuchi, Tsuzuki-ku, Yokohama 224-8558, JAPAN)
}

\begin{abstract}
We have prepared a viscous bicontinuous microemulsion consisting of water/[20 wt\% POE-GIS +30 wt\% PEPTME+47.5 wt\% POE/POP-PDMS + 2.5 wt\% OA)]/DMPS system. A pseudoternary phase diagram was constructed for the mixture, and the bicontinuous microemulsion phase was characterized by means of rheometry and freeze-fracture transmission electron microscopy (FF-TEM).
\end{abstract}

Key words: bicontinuous microemulsion, polymeric silicones, phase behavior, rheometry, freeze-fracture transmission electron microscopy (FF-TEM)

\section{INTRODUCTION}

Microemulsions are thermodynamically stable, isotropic mixtures in which substantial amounts of oil and water (an alternative polar solvent) are brought into a single phase by means of an appropriate surfactant, mostly combined with cosurfactant ${ }^{1-4)}$. Microemulsions, though homogeneous to the eye, are microscopically heterogeneous. Water droplets dispersed in a continuous oil phase are called water-in-oil (W/O) microemulsions, while oil droplets dispersed in a continuous water phase are known as oil-in-water $(\mathrm{O} / \mathrm{W})$ microemulsions. The third type of microemulsion is bicontinuous, in which both oil and water form continuous domain with surfactant residing at extended oil-water interfaces. Among the three, bicontinuous microemulsions have been the subject of extensive research due to great interest in the structure, stability and properties of these systems $^{5-7)}$.

Earlier, the formation and characterization of microemulsion formulations, mostly based on hydrocarbon oils and medium-chain alcohols as cosurfactants, have been studied extensively. Due to low surface tension, excellent spreading capacity on the substrate, and giving a unique dry-lubricant feel to surfaces such as hair and skin, silicone-based oils are gaining much popularity in the cosmetics as skin and body care products ${ }^{8-10)}$. The preparation of $\mathrm{O} / \mathrm{W}$ droplet type microemulsion of polydimethyl-siloxane compounds is extremely difficult task due to spreading tendency of polymeric silicone chain. In the previous paper $^{11)}$, the authors reported the droplet type microemulsion containing POE/POP-PDMS and PEPTME as a cosurfactant in water rich region. To our knowledge, there are no reports of formation of bicontinuous microemulsion of mixed hydrophobic polymeric silicones having very high molecular weight.

In this context, we present a report on the microemulsion which can be formed in a system containing water, POE-GIS, PEPTME, POE/POP-PDMS, OA, and DMPS. The characterization of microemulsion has been done by phase behavior, rheometry and freeze-facture electron microscopy (FF-TEM) technique. We hope the present paper is appealing to the basic understanding of the bicontinuous microemulsion in mixed silicone systems.

\begin{abstract}
Abbreviations: POE-GIS: polyoxyethylene $(20 \mathrm{~mol})$ glycerin isostearate, PEPTME: random copolymer of polyoxyethylene (POE, $38 \mathrm{~mol}$ ) / polyoxypropylene (POP, $10 \mathrm{~mol}$ ) pentaerythritol tetramethyl ether, POE/POP-PDMS: polydimethylsiloxane copolymer of polyoxyethylene (POE, $19 \mathrm{~mol}$ ) / polyoxypropylene (POP, $19 \mathrm{~mol}$ ), OA: oleic acid, DMPS: dimethylpolysiloxane. Chemical structures of these compounds are shown in Scheme 1.
\end{abstract}

*Correspondence to: Reiji Miyahara, Shiseido Research Center, 2-2-1 Hayabuchi, Tsuzuki-ku, Yokohama 224-8558, JAPAN E-mail: reiji.miyahara@to.shiseido.co.jp Accepted August 12, 2008 (received for review June 20, 2008)

Journal of Oleo Science ISSN 1345-8957 print / ISSN 1347-3352 online

http://www.jstage.jst.go.jp/browse/jos/ 


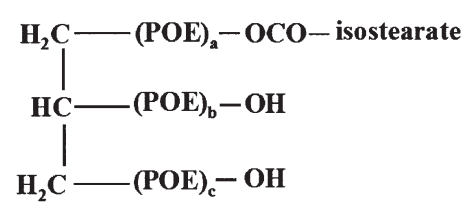

(a)

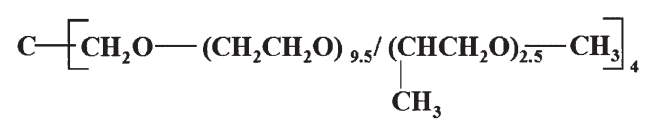

(b)

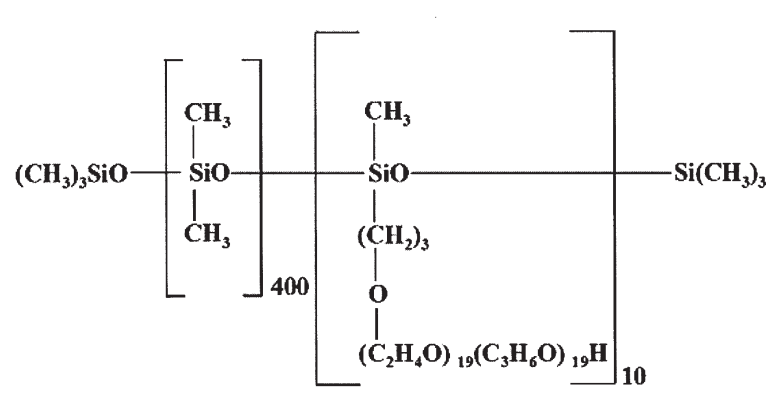

(c)

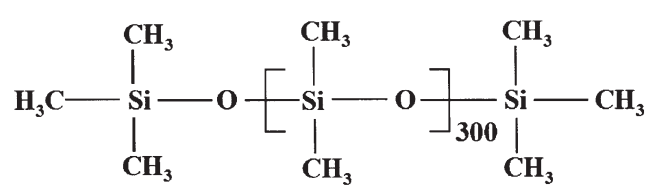

(d)

Scheme 1 Schematic Chemical Structures of POE-GIS (a), PEPTME (b), POE/POP-PDMS (c), and DMPS (d).

\section{EXPERIMENTAL}

\subsection{Materials}

PEPTME was kindly supplied from Nichiyu, Japan. The number average molecular weight of PEPTME is 2300. A detailed structural analysis of PEPTME is presented elsewhere $^{12)}$. POE-GIS and POE/POP-PDMS were purchased from Nikko Chemical Co., and Dow Corning Toray Co. Ltd., Japan, respectively. The number average molecular weight of POE/POP-PDMS is 60000. Finally, OA and DMPS were the products of Nacalai Tesque and Shin-Etsu Chemical Co., Ltd. Japan, respectively. The number average molecular weight of DMPS is 23000. All the chemicals were used as received. Deionized (Millipore filtered) water was used to prepare the samples.

\subsection{Phase diagram}

Samples for phase diagram construction were prepared by weighing the required amounts of reagents into test tubes fitted with screw cap and mixing using a vortex mixer. The samples were kept in a water bath at $25^{\circ} \mathrm{C}$ for equilibration. The phase boundaries were established by observation of turbidity-to-transparency transition and vice versa. Finally, phases were identified by visual observation (through crossed polarizers).

\subsection{Rheometry}

Samples for viscosity measurements were homogenized and kept in a water bath at specified temperature for at least $24 \mathrm{~h}$ to ensure equilibration before performing measurements. The viscosity measurements were performed in a stress-controlled rheometer, AR-G2 (TA Instruments) using cone-plate geometries (diameters of $60 \mathrm{~mm}$ for the low-viscosity sample and $40 \mathrm{~mm}$ for the high-viscosity sample, each with a cone angle of $1^{\circ}$ ) with the plate temperature controlled by Peltier unit. A sample cover provided with the instrument was used to minimize the change in sample composition due to evaporation during the measurement. The zero-shear viscosity $\left(\eta_{\mathrm{o}}\right)$ of the samples was determined from a steady-shear measurement by extrapolating the viscosity-shear-rate curve to zero shear-rate.

\subsection{FF-TEM}

TEM observations of the sample were performed using the freeze-replica method. The sample was rapidly frozen in liquid propane using a cryo preparation system (LEICA EM CPC, LEICA microsystems), and the frozen sample was transferred into a freeze-replica preparing apparatus (FR-7000A, Hitachi Science Systems, Ltd.) and fractured with a glass knife at $-120^{\circ} \mathrm{C}$. A replica film was prepared by evaporating platinum-carbon at $45^{\circ}$ and then carbon at $90^{\circ}$ on the fractured sample. The prepared replica film was washed several times with acetone and distilled water after being taken out of the freeze-replica preparing apparatus and transferred onto a 300 mesh copper grid. The replica thus prepared was observed with a transmission electron microscope (H-7650, Hitachi Science Systems, Ltd.) at an accelerating voltage of $120 \mathrm{kV}$.

\section{RESULTS AND DISCUSSION}

\subsection{Phase behavior}

The pseudoternary phase diagram constructed for water / [20 wt $\%$ POE-GIS + 30 wt $\%$ PEPTME + 47.5 wt $\%$ $\mathrm{POE} / \mathrm{POP}-\mathrm{PDMS}+2.5 \mathrm{wt} \% \mathrm{OA}] / \mathrm{DMPS}$ system at $25^{\circ} \mathrm{C}$ is shown in Fig. 1. The structure of PEPTME resembles with 


\section{0 wt\% POE-GIS + 30 wt\% PEPTME(38/10) + 47.5 wt\% POE/POP-PDMS + 2.5 wt\%OA}

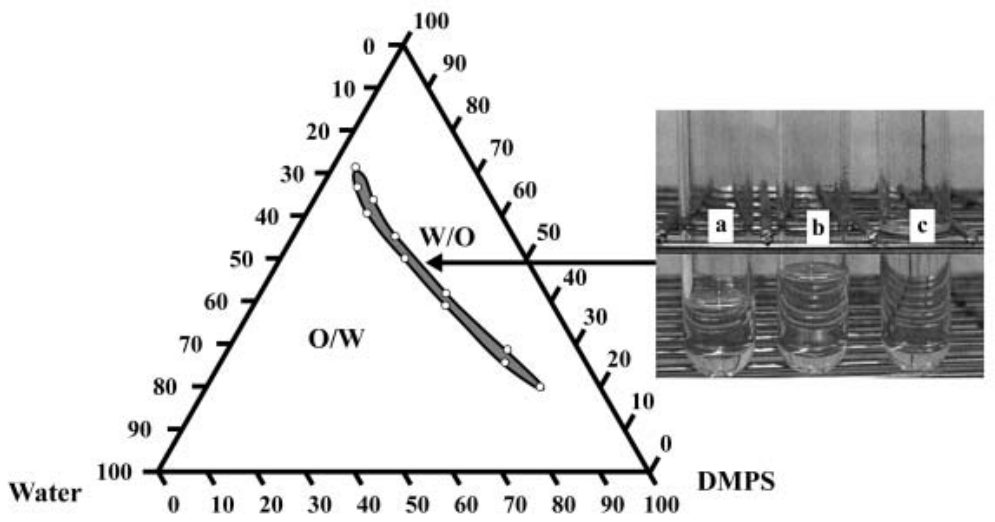

Fig. 1 Pseudoternary Phase Diagram of Water/[20 wt $\%$ POE-GIS +30 $w t \%$ PEPTME + 47.5 wt\% POE/POP-PDMS + $2.5 \mathrm{wt} \% \mathrm{OA}]$ System at $25^{\circ} \mathrm{C}$.

The photographs of microemulsions: wt. fraction of DMPS, $W=$ 0.153 (test tube a), 0.322 (test tube b), and 0.546 (test tube c) in total system along $R=2.33,2.08$, and 1.86 respectively.

the polyoxyethylene chain of polyoxyethylene nonionic surfactants in polairty. PEPTME may thus be considered as a hydrophilic cosurfactant, like polyoxyethylene (POE)/ polyoxypropylene $(\mathrm{POP})$ dimethyl ether $(\mathrm{EPDME})^{13,14)}$, when the concentrations of both PEPTME and polyoxyethylene nonionic surfactants are high, and may possibly be distributed in the polyoxyethylene chain region of the surfactant. In the water / [20 wt\% POE-GIS + $30 \mathrm{wt} \%$ PEPTME+ $47.5 \mathrm{wt} \%$ POE/POP-PDMS + $2.5 \mathrm{wt} \%$ OA] mixture, turbidity was noticed when the water content is less than $\sim 11$ $\mathrm{wt} \%$ in total system. When the amount of water increases from $\sim 11$ to $\sim 19 \mathrm{wt} \%$, a clear isotropic microemulsion is observed. Further increase of water content results into the formation of liquid crystal (not identified) and multiphase regions. In the ternary system, swollen micelles equilibrated with excess of oil $(\mathrm{O} / \mathrm{W})$ and inverted micelles equilibrated with excess of water (W/O) are formed when polymeric silicone oil DMPS is added to low and high [20 wt\% POE-GIS + 30 wt\% PEPTME + 47.5 wt\% POE/POP$\mathrm{PDMS}+2.5 \mathrm{wt} \% \mathrm{OA}] /$ water weight ratio, $R$, respectively. A narrow, highly viscous microemulsion domain extending toward oil corner is formed when $R$ is $\sim 2.33$ to $\sim 1.66$ as indicated by shaded region in the diagram. The microstructure of this microemulsion is thought to be bicontinuous.

\subsection{Rheometry}

To obtain some insight into the microstructure of microemulsion, viscosity measurements were carried out. The plot for zero-shear viscosity for water/[20 wt\% POEGIS + 30 wt $\%$ PEPTME + 47.5 wt $\%$ POE/POP-PDMS + 2.5

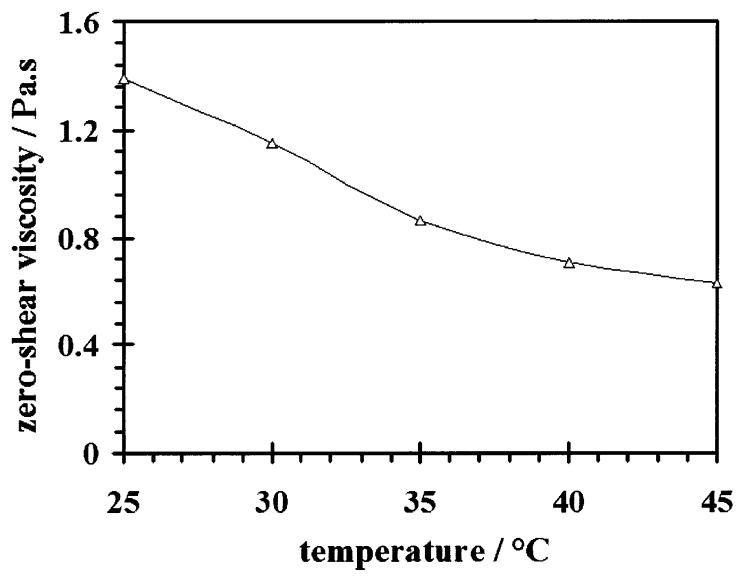

Fig. 2 Variation of Zero-shear Viscosity as a Function Temperature for Water/[20 wt $\%$ POE-GIS $+30 \mathrm{wt} \%$ PEPTME + $47.5 \mathrm{wt} \%$ POE/POP-PDMS + $2.5 \mathrm{wt} \%$ OA]/DMPS System.

The weight fraction of DMPS, $W=0.153$ along $R=$ 2.33. The solid line is only a visual guide.

wt\% OA]/DMPS system as a function of temperature is shown in Fig. 2. As can be seen from Fig. 2, the microemulsion is already very thick, with zero-shear viscosity more than thousand times higher than water at $25^{\circ} \mathrm{C}$. As the temperature increases, the zero-shear viscosity decreases continuously up to $45^{\circ} \mathrm{C}$ suggesting the transition in the shape of aggregates and eventually, phase separation occurs at higher temperature. 


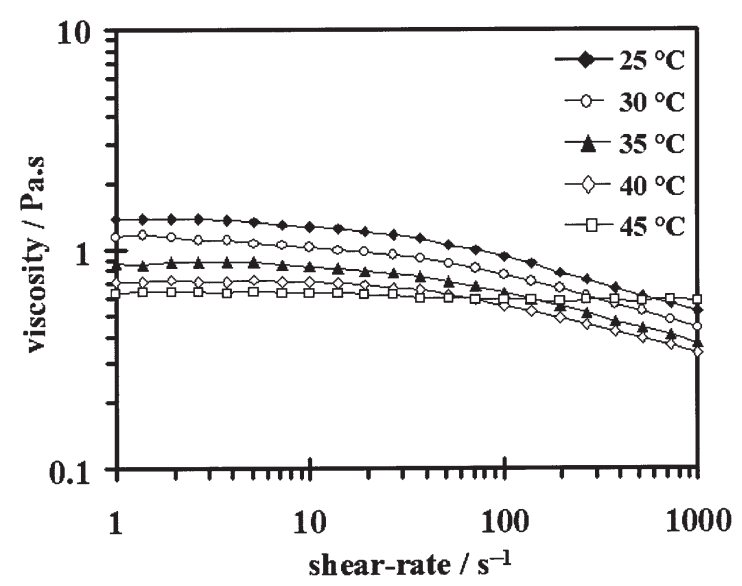

Fig. 3 Steady Shear-rate versus Viscosity Curves for Water / $[20 \mathrm{wt} \%$ POE-GIS + $30 \mathrm{wt} \%$ PEPTME $+47.5 \mathrm{wt} \%$ POE/POP-PDMS $+2.5 \mathrm{wt} \%$ OA]/DMPS System as a Function of Temperature.

The weight fraction of DMPS, $W=0.153$ along $R=$ 2.33. The solid line is only a visual guide.

The steady shear-rate versus viscosity curves for water / [20 wt\% POE-GIS + 30 wt $\%$ PEPTME + 47.5 wt $\%$ $\mathrm{POE} / \mathrm{POP}-\mathrm{PDMS}+2.5 \mathrm{wt} \% \mathrm{OA}] /$ DMPS system as a function of temperature is shown in Fig. 3. At $25^{\circ} \mathrm{C}$, the Newtonian behavior is limited to lower shear rate and viscosity decreases gradually with increasing shear rate. The decrease in viscosity with increasing shear rate corresponds to a shear thinning behavior. With increasing temperature up to $40^{\circ} \mathrm{C}$, the shear thinning behavior is still observed but the viscosity at the lower shear rate decreases, which shows the system is getting less structured. Previous work on microemulsion rheology has indicated that bicontinuous microemulsion exhibits weak shear thinning, particularly if their low-shear viscosities exceed about $10 \mathrm{cP}^{15}$. Referring to this fact, it is suggested that the microemulsion formed at lower temperature is assumed to have a bicontinuous structure. When temperature increases further up to $45^{\circ} \mathrm{C}$, the viscosity is nearly constant almost over the whole range of shear rate studied and the system shows Newtonian behavior suggesting the formation of spherical aggregates (droplet type structures). Probably, as the temperature increases, the dehydration of polyoxyethylene chain takes place and PEPTME shifts closer to the palisade layer of aggregates. Due to shifting of PEPTME, the interfacial curvature of the aggregates increases and forms the globular aggregates, which supports the conclusion derived from viscosity measurement.

\subsection{FF-TEM observation}

In order to make sure of the formation of bicontinuous microemulsion at room temperature $\left(25^{\circ} \mathrm{C}\right)$, FF-TEM mea-

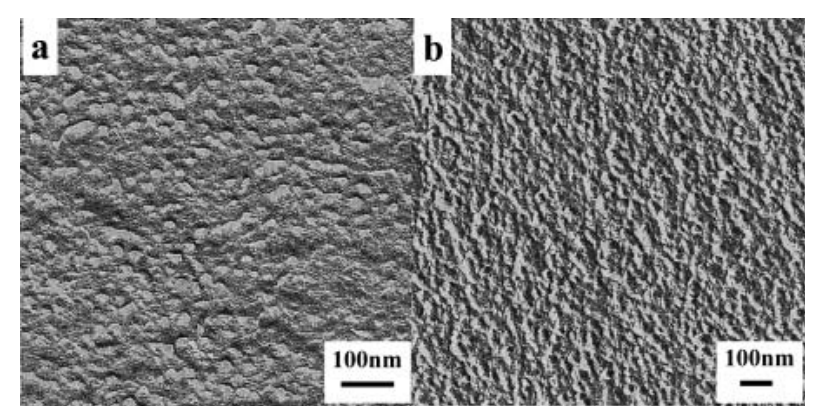

Fig. 4 Freeze-fracture Electron Micrographs of Microemulsions: (a) $W=0.153, R=2.33$, (b) $W=$ $0.322, R=2.08$ for water $/[20 \mathrm{wt} \%$ POE-GIS +30 $\mathrm{wt} \%$ PEPTME $+47.5 \mathrm{wt} \%$ POE/POP-PDMS +2.5 $\mathrm{wt} \%$ OA]/DMPS System.

surements were carried out. The micrographs obtained by FF-TEM technique are presented in Fig. 4. A typical texture of bicontinuous microemulsion can be observed from Fig. 4 (a) for water/[20 wt\% POE-GIS + $30 \mathrm{wt} \%$ PEPTME $+47.5 \mathrm{wt} \% \mathrm{POE} / \mathrm{POP}-\mathrm{PDMS}+2.5 \mathrm{wt} \% \mathrm{OA}] /$ DMPS system at $W=0.153$ along $R=2.33$. However, the micrograph of bicontinuous microstructure of microemulsion shown in Fig. 4 (b) for the same system at $W=0.322$ along $R=2.08$ was not clear. Nevertheless, the result is in well accordance with the viscosity measurement.

\section{CONCLUSION}

In this paper, we have reported the formation of bicontinuous microemulsion in mixed polymeric silicone systems. The phase diagram of the pseudoternary water/[20 $\mathrm{wt} \%$ POE-GIS + $30 \mathrm{wt} \%$ PEPTME + $47.5 \mathrm{wt} \%$ POE/POP$\mathrm{PDMS}+2.5 \mathrm{wt} \% \mathrm{OA}] /$ DMPS system exhibits a narrow viscous bicontinous microemulsion domain in which structural transition from bicontinuous structure to droplet occurs on increasing the temperature. The existence of bicontinuous structure is easily detected by FF-TEM micrographs and viscosity measurements. We believe that the prepared microemulsions using mixed polymeric silicones would be extremely useful in cosmetic fields as skin and hair care products due to unique spreading capacity on the substrate.

\section{ACKNOWLEGEMENT}

S.C.S. is thankful to the Japan Society for the promotion of Science (JSPS) for financial support. 


\section{A Narrow Bicontinuous Microemulsion Domain}

\section{References}

1. Danielsson, I.; Lindman, B. The definition of microemulsion. Colloids Surf. 3, 391-392 (1981).

2. Abe, M.; Yamazaki, T.; Ogino, K.; Kim, Y.J. Phase behavior and physicochemical properties of sodium octyl sulfate/n-decane/1-hexanol/aqueous aluminum chloride middle-phase microemulsion. Langmuir 8, 833-837 (1992).

3. Kunieda, H.; Shinoda, K. Phase behavior and tricritical phenomena in a bile salt system. Bull. Chem. Soc. Jpn. 56, 980-984 (1983).

4. Kunieda, H. Phase behavior and ultralow interfacial tensions around a tricritical point in a sodium taurocholate system. J. Colloid Interface Sci. 116, 224-229 (1987).

5. Lindman, B.; Shinoda, K.; Olsson, U.; Anderson, D.; Karlström, G.; Wennerström, H. On the demonstration of bicontinuous structuresin microemulsions. Colloids Surf. 38, 205-224 (1989).

6. Shinoda, K. Solution behavior of surfactants: The importance of surfactant phase and the continuous change in HLB of surfactant. Prog. Colloid Polym. Sci. 68, 1-7 (1983).

7. Kunieda, H.; Asaoka, H.; Shinoda, K. Two types of surfactant phases and four coexisting liquid phases in a water/nonionic surfactant/triglyceride/hydrocarbon system. J.Phys. Chem. 92, 185-189(1988).

8. Tokuoka, Y.; Uchiyama, H.; Abe, M.; Christian, S.D. Solubilization of some synthetic perfumes by anionic-nonionicmMixed surfactant systems. 1. Langmuir 11, 725729 (1995).

9. Watanabe, K.; Noda, A; Masuda, M.; Nakamura, K.
Bicontinuous microemulsion type cleansing containing silicone oil. I. Comparison of solubilization behavior of cyclic siloxane with polar oils. J. Oleo Sci. 53, 537-546 (2004).

10. Garti, N.; Shevanchman, M.; Shani, A. Formation and investigation of microemulsions based on jojoba oil and nonionic surfactants. J. Am. Oil Chem. Soc. 81, 873877 (2004).

11. Sharma, S.C.; Tsuchiya, K.; Sakai, K.; Sakai, H.; Abe, M.; Komura, S.; Sakamoto, K.; Miyahara, R. Formation and characterization of microemulsion containing polymeric silicone. Langmuir 24, 7658-7662 (2008).

12. Miyahara, R.; Nakanishi, Y.; Maruyama, K.; Sharma, S.C.; Abe, M. Development of novel multifunctional cosmetic raw materials and their applications. IV. The effect of structure of polyoxyethylene/polyoxypropylene derivative on the self -organising structures of surfactants. J. Oleo Sci. in print.

13. Miyahara, R.; Watanabe, K.; Ohmori, T.; Nakama, Y. Development of novel multifunctional cosmetic raw materials and their applications. II. Novel emulsifying method with random copolymer of polyoxyethylene/ polyoxypropylene. J. Oleo Sci. 55, 403- 411 (2006).

14. Miyahara, R.; Watanabe, K.; Ohmori, T.; Nakama, Y. Development of novel multifunctional cosmetic raw materials and their applications. III. Effect of random copolymer of polyoxyethylene/polyoxypropylene on self-organizing structures of nonionic surfactants. $J$. Oleo Sci. 55, 473-482 (2006).

15. Chen, C.-M.; Warr, G.G. Rheology of ternary microemulsions. J. Phys. Chem. 96, 9492-9497 (1992). 